\title{
A Novel Inflammation-Related Prognostic Biomarker for Predicting The Disease-free Survival of Patients With Colorectal Cancer
}

\section{Xiao ling Cai}

Fujian Medical University

\section{Fa Chen}

Fujian Medical University

\section{Li sheng Liang}

Fujian Medical University Union Hospital

\section{Wei Jiang}

Fujian Medical University Union Hospital

\section{Xing Liu}

The First Affiliated Hospital of Fujian Medical University

\section{Dong Wang}

Fujian Medical University Union Hospital

\section{Yun li Wu}

Fujian Medical University

Jin Chen

Fujian Academy of Medical Sciences

\section{Guo xian Guan}

The First Affiliated Hospital of Fujian Medical University

Xian e Peng ( $\square$ peng123456@fjmu.edu.cn )

Fujian Medical University

\section{Research Article}

Keywords: Colorectal cancer, Inflammatory biomarkers, Disease-free Survival, Prognosis, Decision curve analysis

Posted Date: January 24th, 2022

DOI: https://doi.org/10.21203/rs.3.rs-1268811/v1

License: (c) (1) This work is licensed under a Creative Commons Attribution 4.0 International License. Read Full License 
Version of Record: A version of this preprint was published at World Journal of Surgical Oncology on March 11th, 2022. See the published version at https://doi.org/10.1186/s12957-022-02550-0. 


\section{Abstract}

Background: To develop and evaluate the prognostic value of a comprehensive inflammatory biomarker for postoperative colorectal cancer (CRC) patients.

Methods: A total of 646 CRC patients were recruited between August 2017 and December 2019 from Fujian Medical University Union Hospital, with follow-up data up to 2021. The least absolute shrinkage and selection operator method (LASSO) was used to select inflammation indicators in order to construct a comprehensive biomarker (named NSAP). The Cox regression model was utilized to analyze the association between the NSAP and the disease-free survival (DFS) of CRC. Predictive performance and clinical utility of prognostic models were evaluated by area under the curve (AUC) and decision curve analyses (DCAs).

Results: During a median follow-up of 23 months, 95 clinical outcomes were observed, with a one-year survival rate is $89.47 \%$. A comprehensive inflammatory biomarker (NSAP) was established based on four blood indicators (including Neutrophil-to-Lymphocyte Ratio (NLR), Neutrophil×Monocyte-to-Lymphocyte Ratio (SIRI), Albumin-to-Globulin Ratio (AGR) and Platelet-to-Lymphocytes Ratio (PLR)). Patients with a lower NSAP had significantly associated with better DFS of CRC (HR=0.53, 95\% Cl: 0.32-0.89). Moreover, compared to a previously established model, the traditional TNM staging system or/and tumor markers, the nomogram based on NSAP displayed more excellent predictive ability $(0.752$ vs $0.597,0.711$ and $0.735, \mathrm{P}<0.05)$. DCAs also demonstrated that the established nomogram had better utility for decision making.

Conclusions: Our study suggests that NSAP may be a useful comprehensive prognostic biomarker for predicting the DFS of CRC patients. The nomogram based on NSAP can be considered a valuable tool to estimate the prognosis of patients with CRC.

\section{Introduction}

Colorectal cancer (CRC) is the third most common malignant tumor and the second leading cause of death worldwide [1]. To date, the risk factors affecting the prognosis of CRC patients have not yet been fully elucidated. Recurrence and metastasis are dismissed as one of the major prognostic factors for CRC patients [2]. After extended radical surgery, patients presenting with recurrence or metastases have 5-year survival rates of less than $33 \%$ [3-5]. However, there were limited prediction models were developed to foresee the outcomes of recurrence and metastases. Therefore, it is essential to develop a simple and effective predictive model to provide more research evidence for the prognosis of CRC.

Accumulating studies have shown that host immune system may have a dual role in tumor promotion and suppression [6-10]. When the body undergoes the immune response and inflammation, the values and ratios of various indicators change in the blood with different degrees, which may be potentially helpful in outcome prediction $[11,12]$. Previous studies have revealed that several inflammation-related indicators were independent prognostic factors in blood for CRC patients, including the neutrophil-to- 
lymphocyte ratio (NLR), the platelet-to-lymphocyte ratio (PLR), the lymphocyte-to-monocyte ratio (LMR), the albumin-to-globulin ratio (AGR), etc [13-16]. However, most of these studies only involve one or several inflammation-related factors and did not systematically explore the combined effects of inflammatory biomarkers in the prognosis of CRC. Moreover, there have been relatively few studies that focus on the outcomes of recurrence and metastasis.

Therefore, the purpose of this study is to comprehensively explore the roles of various blood indicators in the disease-free survival (DFS) of CRC patients, and to develop a novel inflammation-related comprehensive biomarker using the LASSO algorithm, with a goal to provide research evidence for individual prediction and decision-making.

\section{Materials And Methods}

\section{Study Population}

From August 2017 to December 2019, a total of 646 CRC patients who underwent surgical resection were consecutively enrolled at Fujian Medical University Union Hospital (Fujian, China). Patients were eligible if they met the following criteria: (1) newly pathologically diagnosed with primary CRC; (2) no receipt of any treatment that affected blood indicators; (3) without neoadjuvant radiotherapy or radiochemotherapy prior to surgery. The exclusion criteria were as follows: (1) patients with a history of blood disease or infection and autoimmune disease; (2) patients who suffered from other cancers before being included in the study. All patients provided written informed consent and fully understood the study. The research protocol has been approved by the institutional review board of Fujian Medical University (No.2020KY007) and followed the ethical standards of the Helsinki Declaration.

\section{Data collection}

The patient's demographic and clinical characteristics were abstracted from the hospital medical record system. Next, relevant preoperative laboratory parameters for blood indicator analysis were collected from the tests of blood routine (including neutrophil, lymphocyte, monocyte, high-density lipoprotein counts), blood biochemical (including albumin, globulin counts), and tumor markers including alphafetoprotein (AFP), carcinoembryonic antigen (CEA), carbohydrate antigen 19-9 (CA19-9). Then, eight blood indicators were calculated accordingly: Neutrophil-to-Lymphocyte Ratio (NLR), Lymphocyte-toMonocyte Ratio (LMR), Neutrophil×Monocyte-to-Lymphocyte Ratio (SIRI, Systemic Inflammatory Response Index), Albumin-to-Globulin Ratio (AGR), Platelet-to-Lymphocytes Ratio (PLR), Serum Albumin + 5xtotal Lymphocyte (OPNI/PNI, Onodera's Prognostic Nutritional Index), Monocyte-to-High Density Lipoprotein Ratio (MHR), Monocyte-to-Lymphocyte Ratio (MLR). In addition, we also compared the prognosis of colorectal cancer with newly developed nomogram in previous literatures [17], including tumor size + NLR + PLR + PNI.

\section{Follow-up}


All patients were followed up by trained study interviewers through telephone interview at three months intervals for the first two years postoperatively, and every six months for the next years. DFS was defined as the time from initial surgery to recurrence, metastasis, death (for any reason), or the last follow-up (January 9,2021 ). The overall loss of follow-up rate was $4.3 \%$. During follow-up, 28 cases were dead, 82 patients occurred recurrence and metastasis ( 19 subjects were identified before death), and 551 subjects still alive at the last follow-up.

\section{Statistical Analysis}

The cutoff points for NLR, LMR, SIRI, AGR, PLR, OPNI, MHR, and MLR were determined by the X-tile program (Yale University, Newhaven, Connecticut). Reverse Kaplan-Meier method was used to calculate median follow-up time. A heat map was used to reveal the degree of correlation among the eight blood indicators. Then, the LASSO algorithm was utilized to develop a comprehensive blood indicator. Hazard ratios (HRs) and $95 \%$ confidence intervals ( $\mathrm{Cls}$ ) were estimated based on the univariate and multivariate Cox regression model for exploring independent prognostic factors of CRC. Three prediction models were established using generalized linear model (GLM), receiver operating characteristic (ROC) methods, and the predictive ability were compared through area under the curve (AUC). Decision curve analysis (DCA) was plotted to further assess the clinical usefulness of the models. All statistical analysis was performed using R software (version 4.0.5). P-value less than 0.05 was statistical significance.

\section{Results}

\section{Patients' characteristics}

Of the 646 CRC cases included in the final analysis (Table 1), 403 (62.3\%) were male patients, and 481 (74.46\%) had stage II-III disease. The majority of patients diagnosed with adenocarcinoma (87.46\%) and moderate tumor differentiation (85.91\%). Approximately $10.53 \%-21.12 \%$ of patients tended to have elevated or lowered inflammation-related indicators (Table S1). During a median follow-up time of 23 months, a total of 95 patients had outcome events with a one-year survival rate of $89.47 \%$.

\section{Establishment of a comprehensive blood index}

The heat map provided the correlation between eight blood indicators, reflecting in the area of the circle and the shade of the color (Fig. 1A). Several strong correlations were observed between these indicators, among which SIRI and MLR displayed the strongest correlation strength. Given the correlation between the variables and the limitation of traditional Cox model, the LASSO Cox regression model was used to evaluate the eight selected indicators (Fig. 1B and Fig. S1). The formula for the comprehensive blood index was obtained as follows: NSAP $=(0.01567 \times \mathrm{NLR})+(0.01733 \times \mathrm{SIRI})+(0.23396 \times \mathrm{AGR})+$ (0.00299 $\times$ PLR). According to this formula, 646 patients were then divided into two groups using the optimal cut-point determined by the X-Tile software: the high-NSAP group (>1.05) and the low-NSAP group ( $\leq 1.05)$. 
Prognostic value of NSAP compared with their higher counterparts, patients with a lower NSAP tended to have a significantly better DFS $(P<0.001$, Fig. 2). The univariate and multivariate Cox models were both showed that the high-NSAP was associated with an increased risk of DFS: The HRs were 2.56 (95\% Cl: 1.64-4.00, $\mathrm{P}<0.001)$, and 1.89 (95\% Cl: 1.12-3.13, $\mathrm{P}=0.015)$, respectively. Additionally, tumor stage (IV vs I stage, HR: 4.92, 95\% Cl: 1.62-14.97), AFP (<20 vs $\geq 20$, HR: 0.53, 95\% Cl: 0.33-0.85), CEA (<5 vs $\geq 5$, HR: $0.53,95 \% \mathrm{Cl}: 0.33-0.88)$ were found to be independent prognostic factors for DFS in the multivariate analysis (Table 2 and Fig. S3).

Next, a prognostic nomogram based on the NSAP and other three factors selected by multivariate analysis was developed to quantitatively predict 1-year, 2-year and 3-year DFS (Fig. 3). The predictive ability of established nomogram (Model-3) was superior to that of the Model-1 (TNM staging system), the Model-2 (TNM staging system + AFP + CEA) and Model-4 (Tumor size + NLR + PLR + OPNI). The AUC were 0.752 (95\% Cl: 0.717-0.785), 0.711 (95\% Cl: 0.674-0.746), 0.735 (95\% Cl: 0.699-0.768), 0.592 (95\% Cl: 0.558-0.635), respectively (Fig. 4A). For internal validation, calibration curves for predicting 1-year and 2-year DFS indicated a good match between the predicted probabilities and the actual observations (Fig. S4). In addition, DCA revealed similar results that the established nomogram had more positive net benefit than previously established prognostic nomograms, traditional TNM staging system or/and tumor biomarkers (Fig. 4B).

\section{Discussion}

To the best of our knowledge, the present study is one of few studies to establish a comprehensive blood index (NSAP) based on a combination of four inflammation-related parameters using the LASSO method. Our study demonstrated that NSAP was an independent prognostic factor for CRC, and the high-NSAP was associated with poor DFS. The nomogram based on NSAP and the TNM staging system and tumor markers displayed a high predictive performance and clinical decision value.

A large number of studies have shown that systemic inflammation has become an essential part of tumorigenesis, proliferation, survival and migration, and is likely to become a new direction for cancer treatment and monitoring [18-20]. To date, several hematological inflammation markers (NLR, OPNI, SIRI, PLR, LMR, AGR) were reported to have potential prognostic values in multiple tumor types [21-26]. In this study, we also found AFP and CEA were valuable independent factors for CRC prognosis, which is supported by results from several previous studies [27-28]. Of note, except for the above six hands, we also added two additional inflammation indexes (MHR and MLR), which have not yet fully proven the predictive value in the prognosis of CRC.

Although a single inflammatory index was proved to have predictive ability, the comprehensiveness and accuracy are not satisfactory. In the current study, varying degrees of correlation among the eight indicators were also observed. Therefore, we employed the LASSO method to solve these problems instead of the traditional Cox model. The most prominent advantage of LASSO algorithm is that the relatively unimportant coefficients of independent variables become 0 and are excluded from modeling 
through penalized regression on all variable coefficients [29]. It is particularly suitable for linear models with a reduced number of parameters, selection of parameters, and estimation of sparse parameters. Relevant studies have verified that the LASSO model is beneficial to improving the accuracy of the prediction model $[29,30]$. In this study, using the LASSO method, we developed a novel comprehensive inflammation index (NSAP), which may be a useful and valuable index for predicting the survival prognosis of patients after CRC operation. In addition, compared with the model established by quoting the article, the model established by us has better predictive performance.

It is not yet clear that the exact underlying mechanism of the close correlation between the high-NSAP and the poor DFS. We found that three of the four indicators included in NSAP are related to lymphocyte count, except for AGR. Previous studies showed that local control of metastatic invasion by the immune system might be critical to survival. The presence of lymphocytes in the tumor may be a favorable prognostic sign [31]. For example, memory $T$ cells in colorectal cancer can change tumor matrix or tumor cells in the adaptive immune response to reduce the metastatic potential of tumor cells. It may be that the transport characteristics, density, and long-term anti-tumor ability of T cells play a central role in controlling tumor recurrence [32]. Similarly, in solid tumors, tumor infiltrating lymphocytes show oligoclonal expansion, recognition of tumor antigens, and tumor-specific cytolytic activity in vitro, which are conducive to improving clinical results, including delayed recurrence or delayed death [33,34]. Additionally, the globulin in AGR, the main protein produced by immune organs, could reflect the body's inflammation and immune status [35]. Therefore, these indicators have the potential to predict the survival of patients with CRC after surgery.

Nevertheless, the current research has some limitations that should be considered. First of all, although we have collected complete follow-up data of 646 patients, the findings of this study were still limited by the lack of external verification and a relatively small sample size. Second, as a single-center study, it will inevitably bring about potential selection bias. Future multi-center studies with larger sample sizes are warranted to confirm our findings further. Third, the three-year follow-up time has affected the tracking of more outcome events and limited the observation of the survival rate of CRC for a longer time. It is necessary to continue the follow-up visit according to the established follow-up plan in the future.

\section{Conclusion}

This study developed a novel comprehensive inflammatory biomarker (NSAP) and suggested that NSAP is strongly associated with the DFS in CRC patients. Compared with the previously established model, the traditional TNM staging system and tumor markers, NASP can effectively improve the predictive ability of the prognostic model. More prospective multi-center data sets are still needed to confirm our findings and the practicality of clinical decision-making in the future.

\section{Abbreviations}


CRC: Colorectal cancer; LASSO: The least absolute shrinkage and selection operator method; NSAP: A comprehensive biomarker; DFS: The disease-free survival; AUC: Area under the curve; DCAs: Decision curve analyses; AFP: Alpha-fetoprotein; CEA: Carcinoembryonic antigen; CA19-9: Carbohydrate antigen 19-9; NLR: Neutrophil-to-Lymphocyte Ratio; LMR: Lymphocyte-to-Monocyte Ratio; SIRI: Systemic Inflammatory Response Index; AGR: Albumin-to-Globulin Ratio; PLR: Platelet-to-Lymphocytes Ratio; OPNI/PNI: Onodera's Prognostic Nutritional Index; MHR: Monocyte-to-High Density Lipoprotein Ratio; MLR: Monocyte-to-Lymphocyte Ratio; HRs: Hazard ratios; Cls: Confidence intervals; GLM:Generalized linear model; ROC: Receiver operating characteristic.

\section{Declarations}

\section{Acknowledgements}

None.

\section{Author's contributions}

CXL, CF and LLS were responsible for obtaining and analyzing data, drafting manuscripts and making critical revisions. JWZ, LX, WD, WYL and CJY mainly were responsible for technical support, relevant references and manuscripts' structure and grammar. GGX and PXE were responsible for the conception, design and review.

\section{Funding}

This study was funded by Science Foundation of the Fujian Province (No.2019J0105), Special Financial Foundation of Fujian Provincial (No.2020B1050), Young and Middle-aged Backbone Training Project in the Health System of Fujian province (2015-ZQN-JC-17), and the Talent Programs granted from The First Affiliated Hospital of Fujian Medical University, the Talents Training Project for the Key Young Scholars by Fujian Provincial Health Commission of China (2016-ZQN-22).

\section{Data availability}

The datasets used and/or analyzed during the current study are available from the corresponding author upon reasonable request.

\section{Ethics approval and consent to participate}

The research protocol has been approved by the institutional review board of Fujian Medical University (No.2020KY007) and followed the ethical standards of the Helsinki Declaration.

\section{Consent for publication}

Not applicable. 


\section{Competing interests}

The authors have declared that no competing interest exists.

\section{References}

1. Bray F, Ferlay J, Soerjomataram I, Siegel RL, Torre LA, Jemal A. Global cancer statistics 2018 : GLOBOCAN estimates of incidence and mortality worldwide for 36 cancers in 185 countries. CA Cancer J Clin. 2018;68:394-424.

2. Mahvi DA, Liu R, Grinstaff MW, Colson YL, Raut CP. Local Cancer Recurrence: The Realities, Challenges, and Opportunities for New Therapies. CA Cancer J Clin. 2018;68:488-505.

3. Yamada K, Ishizawa T, Niwa K, Chuman Y, Aikou T. Pelvic exenteration and sacral resection for locally advanced primary and recurrent rectal cancer. Dis Colon Rectum. 2002;45:1078-84.

4. Shen F, Zhu Y, Wang F, Cai X, Ding H, Zhou F, et al. Clinical significance of circulating tumour cells and tumour marker detection in the chemotherapeutic evaluation of advanced colorectal cancer. Colorectal Dis. 2021;10.1111/codi.15939.

5. Hahnloser D, Nelson H, Gunderson LL, Hassan I, Haddock MG, O'Connell MJ, et al. Curative potential of multimodality therapy for locally recurrent rectal cancer. Ann Surg. 2003;237:502-8.

6. Song M, Chan AT. The Potential Role of Exercise and Nutrition in Harnessing the Immune System to Improve Colorectal Cancer Survival. Gastroenterology. 2018;155:596-600.

7. Hurtado CG, Wan F, Housseau F, et al. Roles for Interleukin 17 and Adaptive Immunity in Microenvironment of Colorectal Cancer to Drive Poor-Prognosis Subtypes and Metastasis. Cancer Cell. 2019;36:319-336.e7.

8. Jackstadt R, van Hooff SR, Leach JD, et al. Epithelial NOTCH Signaling Rewires the Tumor Microenvironments in Metastases and the Survival of Colorectal Cancer Patients. Cancer Cell. 2019;36(3):319-336.e7.

9. Van den Eynde M, Mlecnik B, Bindea G, Cortes-Lavaud X, Lohuis JO, Ridgway RA, et al. The Link between the Multiverse of Immune Microenvironments in Metastases and the Survival of Colorectal Cancer Patients. Cancer Cell. 2018;34:1012-1026.e3.

10. Song M, Nishihara R, Wang M, Chan AT, Qian ZR, Inamura K, et al. Plasma 25-hydroxyvitamin D and colorectal cancer risk according to tumour immunity status. Gut. 2016;65:296-304.

11. Cannon NA, Meyer J, lyengar P, Ahn C, Westover KD, Choy H, et al. Neutrophil-lymphocyte and platelet-lymphocyt ratios as prognostic factors after stereotactic radiation therapy for early-stage non-small-cell lung cancer. J Thorac Oncol. 2015;10:280-5. 
12. Yodying H, Matsuda A, Miyashita M, Matsumoto S, Sakurazawa N, Yamada M, et al. Prognostic Significance of Neutrophil-to-Lymphocyte Ratio and Platelet-to-Lymphocyte Ratio in Oncologic Outcomes of Esophageal Cancer: A Systematic Review and Meta-analysis. Ann Surg Oncol. 2016;23:646-54.

13. Dong YW, Shi YQ, He LW, Su PZ. Prognostic significance of neutrophil-to-lymphocyte ratio in rectal cancer: a meta-analysis. Onco Targets Ther. 2016;9:3127-34.

14. Guo G, Wang Y, Zhou Y, Quan Q, Zhang Y, Wang H, et al. Immune cell concentrations among the primary tumor microenvironment in colorectal cancer patients predicted by clinicopathologic characteristics and blood indexes. J Immunother Cancer. 2019;7:179.

15. Chan JC, Chan DL, Diakos Cl, Engel A, Pavlakis N, Gill A, et al. The Lymphocyte-to-Monocyte Ratio is a Superior Predictorof Overall Survival in Comparison to Established Biomarkers of Resectable Colorectal Cancer. Ann Surg. 2017;265:539-546.

16. Li Y, Jia H, Yu W, Xu Y, Li X, Li Q, et al. Nomograms for predicting prognostic value of inflammatory biomarkers in colorectal cancer patients after radical resection. Int J Cancer. 2016;139:220-31.

17. Sun Y, Huang Z, Chi P. An inflammation index-based prediction of treatment response to neoadjuvant chemoradiotherapy for rectal mucinous adenocarcinoma. Int J Clin Oncol. 2020;25(7):1299-1307.

18. Coussens LM, Werb Z. Inflammation and cancer. Nature. 2002;420:860-7.

19. Mantovani A, Allavena P, Sica A, Balkwill F. Cancer-related inflammation. Nature. 2008;454:436-44.

20. Balkwill F, Mantovani A. Inflammation and cancer: back to Virchow? Lancet. 2001;357:539-45.

21. Lin J, Chen L, Chen Q, Zhuang Z, Bao X, Qian J, et al. Prognostic value of preoperative systemic inflammation response index in patients with oral squamous cell carcinoma: Propensity score-based analysis. Head Neck. 2020;42:3263-3274.

22. Zhuang Z, Li Y, Hong Y, Chen L, Qian J, Lin J, et al. A novel prognostic score based on systemic inflammatory biomarkers for patients with oral squamous cell carcinoma. Oral Dis. 2021;10.1111/odi.13774.

23. Choi Y, Kim JW, Nam KH, Han SH, Kim JW, Ahn SH, et al. Systemic inflammation is associated with the density of immune cells in the tumor microenvironment of gastric cancer. Gastric Cancer. 2017;20:602-611.

24. Zhang H, Shang X, Ren P, Gong L, Ahmed A, Ma Z, et al. The predictive value of a preoperative systemic immune-inflammation index and prognostic nutritional index in patients with esophageal squamous cell carcinoma. J Cell Physiol. 2019;234:1794-1802.

25. Zheng L, Zou K, Yang C,Chen F, Guo T, Xiong B. Inflammation-based indexes and clinicopathologic features are strong predictive values of preoperative circulating tumor cell detection in gastric cancer 
patients. Clin TransI Oncol. 2017;19:1125-1132.

26. Pedrazzani C, Mantovani G, Fernandes E, Bagante F, Luca Salvagno G, Surci N, et al. Assessment of neutrophil-to-lymphocyte ratio, platelet-to-lymphocyte ratio and platelet count as predictors of longterm outcome after R0 resection for colorectal cancer. Sci Rep. 2017;7:1494.

27. Ren F, Weng W, Zhang Q, Tan C, Xu M, Zhang M, et al. Clinicopathological features and prognosis of AFP-producing colorectal cancer: a single-center analysis of 20 cases. Cancer Manag Res. 2019;11:4557-4567.

28. Xu Z, Wang H, Gao L, Zhang H, Wang X. YAP Levels Combined with Plasma CEA Levels Are Prognostic Biomarkers for Early-Clinical-Stage Patients of Colorectal Cancer. Biomed Res Int. 2019;2019:2170830.

29. Robert T. Regression shrinkage and selection via the Lasso. J. R. Stat. Soc.Ser. B Methodol. 1996;58:267-28.

30. Wang JB, Li P, Liu XL, Zheng QL, Ma YB, Zhao YJ, et al. An immune checkpoint score system for prognostic evaluation and adjuvant chemotherapy selection in gastric cancer. Nat Commun. 2020;11:6352.

31. Reichling C, Taieb J, Derangere V, Klopfenstein Q, Le Malicot K, Gornet JM, et al. Artificial intelligenceguided tissue analysis combined with immune infiltrate assessment predicts stage III colon cancer outcomes in PETACC08 study. Gut. 2020;69:681-690.

32. Pagès $F$, Berger $A$, Camus $M$, et al. Effector memory $T$ cells, early metastasis, and survival within human colorectal tumors predict clinical outcome. Science. 2006;313:1960-4.

33. Galon J, Costes A, Sanchez-Cabo F, Kirilovsky A, Mlecnik B, Lagorce-Pagès C, et al. Type, density, and location of immune cells within human colorectal tumors predict clinical outcome. Science. 2006;313:1960-4.

34. Zhang L, Conejo-Garcia JR, Katsaros D, Gimotty PA, Massobrio M, Regnani G, et al. Intratumoral T cells, recurrence, and survival in epithelial ovarian cancer. N Engl J Med. 2003;348:203-13.

35. Ballow M. Mechanisms of action of intravenous immune serum globulin in autoimmune and inflammatory diseases. J Allergy Clin Immunol. 1997;100:151-7.

\section{Tables}


Table 1 Baseline clinicopathological characteristics of patients with colorectal cancer

\begin{tabular}{|c|c|c|}
\hline Variables & No. of patients(\%) & No. of outcome(\%) \\
\hline \multicolumn{3}{|l|}{ Gender } \\
\hline Female & $243(37.62)$ & $36(37.89)$ \\
\hline Male & $403(62.38)$ & $59(62.11)$ \\
\hline \multicolumn{3}{|l|}{ Age } \\
\hline$\geq 60$ & $379(58.67)$ & $53(55.79)$ \\
\hline$<60$ & $267(41.33)$ & $42(42.21)$ \\
\hline \multicolumn{3}{|c|}{ Adjuvant therapy } \\
\hline No & $248(38.39)$ & $28(29.47)$ \\
\hline Chemotherapy & $376(58.20)$ & $63(66.32)$ \\
\hline Other ${ }^{a}$ & $22(3.41)$ & $4(4.21)$ \\
\hline \multicolumn{3}{|l|}{ BMI } \\
\hline$\geq 24$ & 198(30.65) & $28(29.47)$ \\
\hline$<18.5$ & $40(6.19)$ & $5(5.26)$ \\
\hline $18.5-23.9$ & $408(63.16)$ & $62(65.27)$ \\
\hline \multicolumn{3}{|l|}{ Smoking } \\
\hline No & 602(93.19) & 83(87.37) \\
\hline Yes & $44(6.81)$ & $12(12.63)$ \\
\hline \multicolumn{3}{|l|}{ Drinking } \\
\hline No & $462(71.5)$ & $68(71.58)$ \\
\hline Yes & $184(28.5)$ & $27(28.42)$ \\
\hline \multicolumn{3}{|l|}{ Comorbidity } \\
\hline No & $310(47.99)$ & $46(48.42)$ \\
\hline Yes & $336(52.01)$ & $49(51.58)$ \\
\hline \multicolumn{3}{|c|}{ Family history of cancer } \\
\hline No & $515(79.72)$ & $75(78.95)$ \\
\hline Yes & $131(20.28)$ & $20(21.05)$ \\
\hline Tumor stage & & \\
\hline
\end{tabular}




\begin{tabular}{lll} 
I & $120(18.57)$ & $8(8.42)$ \\
\hline II & $233(36.07)$ & $19(20.00)$ \\
\hline III & $248(38.39)$ & $39(41.05)$ \\
\hline IV & $45(6.97)$ & $29(30.53)$
\end{tabular}

Specimen type

\begin{tabular}{lll} 
Raised type & $236(36.53)$ & $26(27.37)$ \\
\hline Ulcer type & $362(56.04)$ & $52(54.74)$ \\
\hline Other $^{b}$ & $48(7.43)$ & $17(17.89)$
\end{tabular}

Tumor differentiation

\begin{tabular}{lll}
\hline Well & $19(2.94)$ & $3(3.16)$ \\
\hline Moderate & $555(85.91)$ & $73(76.84)$ \\
\hline Poor & $33(5.11)$ & $12(12.63)$ \\
\hline Undifferentiated & $39(6.04)$ & $7(7.37)$ \\
\hline
\end{tabular}

Histological classification

\begin{tabular}{|c|c|c|}
\hline Adenocarcinoma & $565(87.46)$ & $82(86.32)$ \\
\hline Mucinous carcinoma and other ${ }^{c}$ & $81(12.54)$ & 13(13.68) \\
\hline
\end{tabular}

Tumor site

\begin{tabular}{lll}
\hline Colon & $337(52.17)$ & $58(61.05)$ \\
\hline Rectum & $281(43.50)$ & $33(34.74)$ \\
\hline Other $^{d}$ & $28(4.33)$ & $4(4.21)$ \\
\hline Lymph node metastasis & & \\
\hline No & $386(59.75)$ & $38(40.00)$ \\
\hline Yes & $260(40.25)$ & $57(60.00)$ \\
\hline
\end{tabular}

Tumor size

$\begin{array}{lll}\geq 3 \mathrm{~cm} & 460(71.21) & 75(78.95) \\ <3 \mathrm{~cm} & 186(28.79) & 20(21.05)\end{array}$

Abbreviation: BMI, body mass index;

Other ${ }^{\mathrm{a}}$ included radiotherapy or chemoradiotherapy;

Other ${ }^{\mathrm{b}}$ included superficial type, undifferentiated type and ulcer infiltrating type; 
Other ${ }^{\mathrm{C}}$ included undifferentiated carcinoma;

Other ${ }^{d}$ included cecum, junction of rectum and sigmoid colon and multi-site tumors. 
Table 2 Univariate and multivariate analysis for disease-free survival

\begin{tabular}{|c|c|c|c|c|}
\hline & Univariate analysis & & Multivariate anal & \\
\hline Variables & $\mathrm{HR}(95 \% \mathrm{Cl})$ & $\mathrm{P}$ & $\mathrm{HR}(95 \% \mathrm{Cl})$ & $\mathrm{P}$ \\
\hline Gender(Male vs Female) & $1.01(0.66-1.52)$ & 0.975 & $0.97(0.60-1.57)$ & 0.905 \\
\hline Age(years, $<60$ vs $\geq 60$ ) & $1.29(0.86-1.94)$ & 0.213 & $1.56(0.97-2.52)$ & 0.069 \\
\hline Adjuvant therapy & & & & \\
\hline No & Ref. & - & Ref. & - \\
\hline Chemotherapy & $1.49(0.95-2.32)$ & 0.082 & $1.12(0.63-1.98)$ & 0.702 \\
\hline Other & $1.70(0.60-4.86)$ & 0.320 & $1.12(0.35-3.57)$ & 0.845 \\
\hline BMI & & & & \\
\hline$\geq 24$ & Ref. & - & Ref. & - \\
\hline$<18.5$ & $0.90(0.35-2.33)$ & 0.828 & $1.59(0.58-4.38)$ & 0.368 \\
\hline $18.5-23.9$ & $1.09(0.69-1.70)$ & 0.719 & $1.23(0.74-2.02)$ & 0.426 \\
\hline Smoking(Yes vs No) & $1.11(0.60-2.05)$ & 0.734 & $1.09(0.56-2.11)$ & 0.809 \\
\hline Drinking(Yes vs No) & $0.96(0.61-1.50)$ & 0.849 & $1.16(0.69-1.93)$ & 0.576 \\
\hline Comorbidity(Yes vs No) & $1.07(0.71-1.60)$ & 0.744 & $1.22(0.78-1.93)$ & 0.381 \\
\hline Family history of cancer(Yes vs No) & $0.80(0.49-1.31)$ & 0.380 & $0.79(0.47-1.34)$ & 0.377 \\
\hline Tumor stage & & & & \\
\hline I & Ref. & - & Ref. & - \\
\hline II & $1.35(0.59-3.10)$ & 0.473 & $0.80(0.32-2.01)$ & 0.641 \\
\hline III & $2.60(1.21-5.58)$ & 0.014 & $1.04(0.35-3.13)$ & 0.942 \\
\hline IV & $15.70(7.09-34.56)$ & $<0.001$ & $4.91(1.61-15.03)$ & 0.005 \\
\hline Specimen type & & & & \\
\hline Raised type & Ref. & - & Ref. & - \\
\hline Ulcer type & $1.50(0.94-2.41)$ & 0.090 & $1.14(0.68-1.90)$ & 0.619 \\
\hline Other & $3.94(2.14-7.27)$ & $<0.001$ & $1.82(0.90-3.69)$ & 0.098 \\
\hline Tumor differentiation & & & & \\
\hline Well & Ref. & - & Ref. & - \\
\hline Moderate & $1.21(0.38-3.86)$ & 0.747 & $0.53(0.15-1.80)$ & 0.306 \\
\hline
\end{tabular}




\begin{tabular}{lllll} 
Poor & $4.96(1.31-16.81)$ & 0.018 & $1.39(0.33-5.74)$ & 0.653 \\
\hline Undifferentiated & $1.96(0.50-7.65)$ & 0.333 & $1.09(0.25-4.77)$ & 0.911 \\
\hline Histological classification & & & & \\
\hline Adenocarcinoma & Ref. & - & Ref. & - \\
\hline Mucinous carcinoma and other & $1.22(0.68-2.19)$ & 0.512 & $0.48(0.23-1.03)$ & 0.059 \\
\hline Tumor site & & & & \\
\hline Colon & Ref. & - & Ref. & - \\
\hline Rectum & $0.70(0.46-1.07)$ & 0.103 & $1.07(0.66-1.73)$ & 0.794 \\
\hline Other & $0.85(0.31-2.34)$ & 0.753 & $1.19(0.41-3.47)$ & 0.748 \\
\hline Lymph node metastasis(Yes vs No) & $2.63(1.74-3.98)$ & $<0.001$ & $1.93(0.94-3.95)$ & 0.073 \\
\hline Tumor size $(\geq 3$ vs $<3)$ & $0.69(0.42-1.13)$ & 0.144 & $0.73(0.42-1.27)$ & 0.261 \\
\hline AFP $(<20$ vs $\geq 20)$ & $0.58(0.37-0.90)$ & 0.016 & $0.53(0.33-0.86)$ & 0.010 \\
\hline CEA $(<5$ vs $\geq 5)$ & $0.41(0.27-0.63)$ & $<0.001$ & $0.54(0.33-0.89)$ & 0.015 \\
\hline CA199 $(<37$ vs $\geq 37)$ & $0.40(0.26-0.62)$ & $<0.001$ & $0.93(0.55-1.58)$ & 0.786 \\
\hline NSAP $(\leq 1.05$ vs $>1.05)$ & $0.39(0.25-0.61)$ & $<0.001$ & $0.53(0.32-0.87)$ & 0.013
\end{tabular}

Abbreviation: AFP, alpha-fetoprotein; CEA, carcinoembryonic antigen; CA19-9, carbohydrate antigen 199; NSAP (comprehensive blood index), including LMR, SIRI, AGR and PLR; $p<0.05$.

\section{Figures}

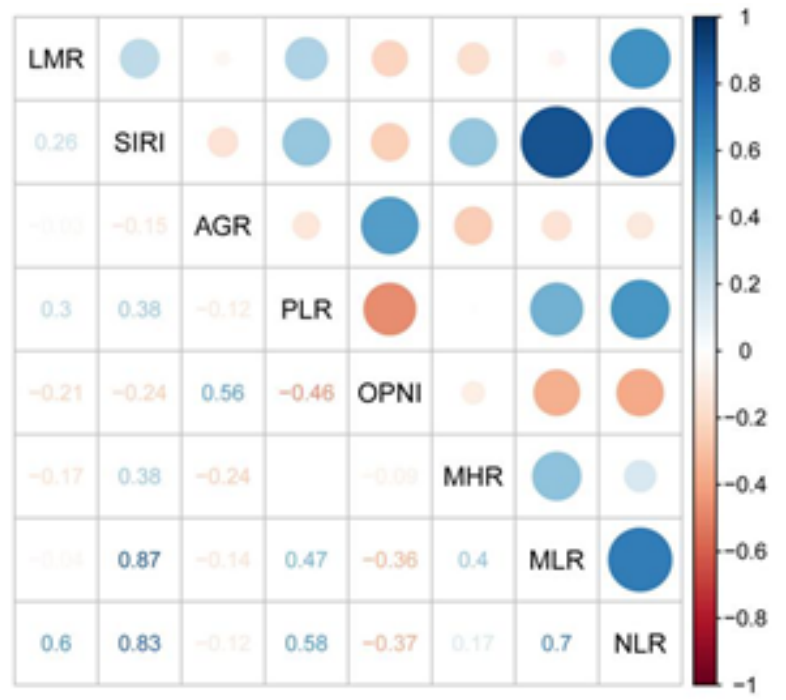

(A)

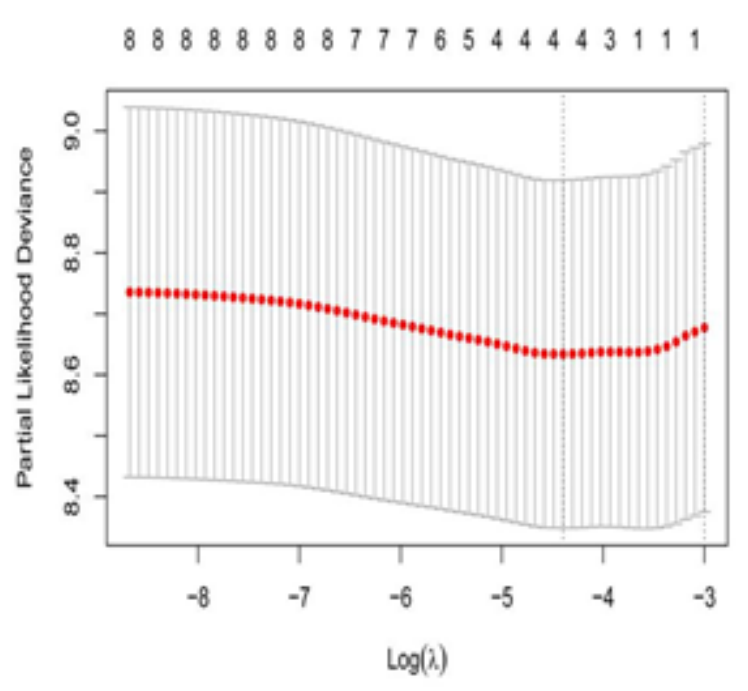

(B)

Figure 1 
A Correlation heat map of inflammation indicators. B Partial likelihood deviance of the LASSO analysis.

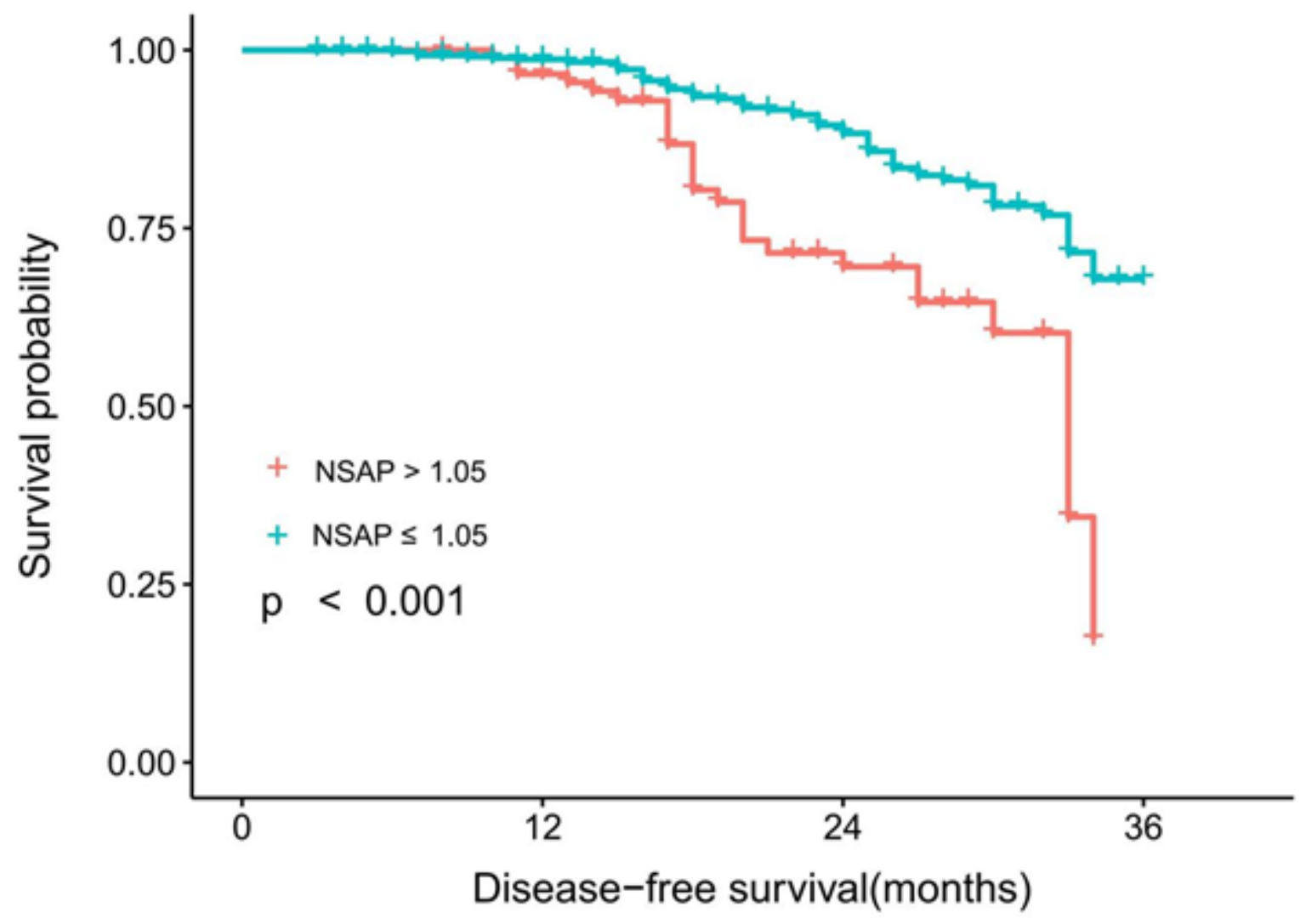

Figure 2

Survival curve of inflammation comprehensive index. 
Points

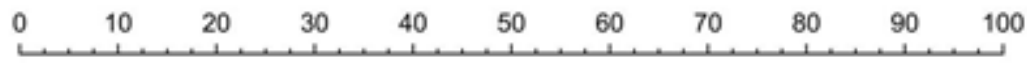

Tumor stage

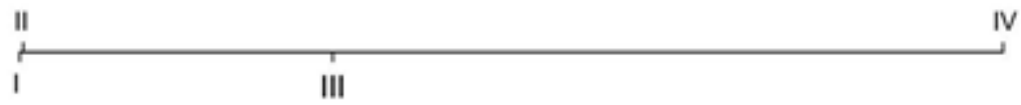

AFP

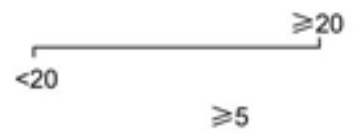

CEA

NSAP
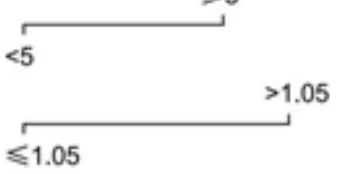

Total Points

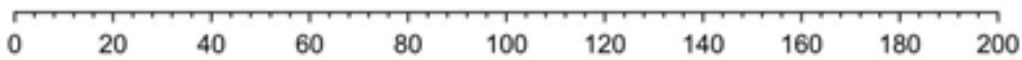

1-year survival

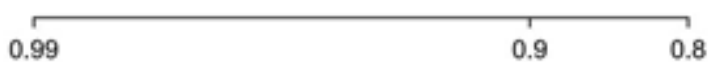

2-year survival

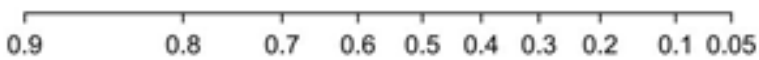

3-year survival

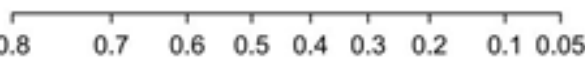

\section{Figure 3}

Nomogram composed of Tumor stage, AFP, CEA, and NSAP.

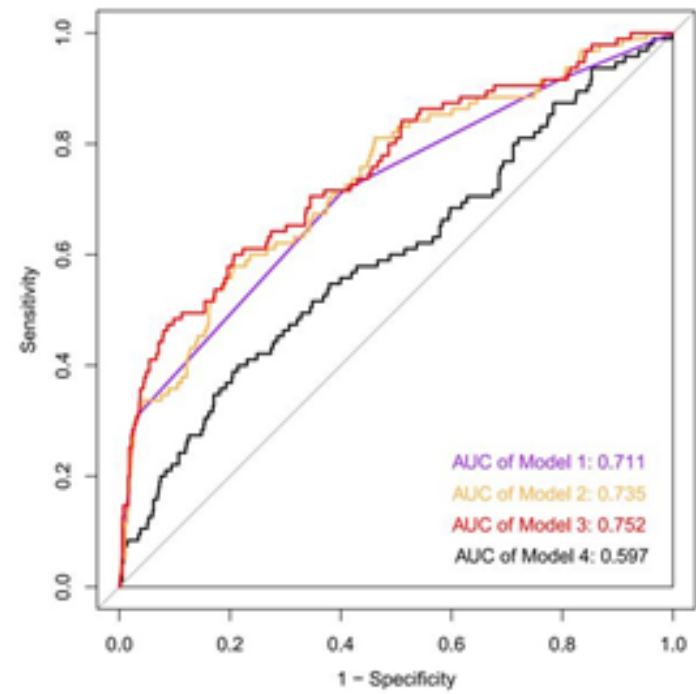

(A)

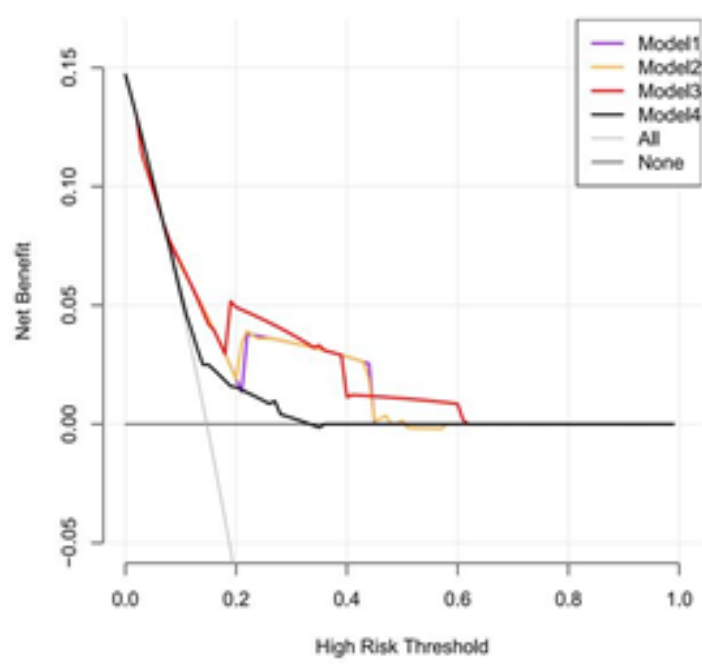

(B)

Figure 4 
A Comparison of AUC between four models. B Comparison of DCA between four models.

\section{Supplementary Files}

This is a list of supplementary files associated with this preprint. Click to download.

- Supportingmaterial.docx 